$$
\text { "mader" — 2008/7/31 — 11:38 — page } 15 \text { - \#1 }
$$

\title{
Heads or Tails gambling — what can be learned about probability?
}

\author{
Attilla MÁder
}

Abstract. During the teaching of probability theory, a problem may appear whose solution requires the use of methods that are unfamiliar to secondary school students. In this paper, examples of methods that can resolve this difficulties are demonstrated, which could in future allow school students to tackle and solve a wide variety of problems involving probability.

Key words and phrases: probability, teaching probability, heads or tails games, infinite series.

ZDM Subject Classification: E53, E54, I34, I35, K53, K54, R24.

\section{Introduction}

During the past few years in secondary education special emphasis has been laid on probability theory. The upper-level curriculum requires elements of geometric probability theory, the concept of a variable and some discrete probability distributions, their expectation values, and variance as well the classical probability theory. The graphical conceptual understanding of the law of large numbers is among the curriculum requirements as well. It is not surprising therefore that this extended curriculum has resulted in the occurrence of unfamiliar problems.

In lessons on probability theory students often say they want to calculate the probability of winning in the games they play. This kind of demand is somewhat self-evident, since why would we study probability theory if we cannot use it in our everyday life? The above play an essential role in the new, upper level maturity 


$$
\text { "mader" — 2008/7/31 — 11:38 — page } 16-\# 2
$$

exam. Actually, the emphasis has not been on how to use these methods in other sciences, but rather on how to solve problems taken from real life. The socalled realistic teaching of mathematics separates practical tasks from the abstract problems of theoretical education. The Freudenthal method, which originated from the Netherlands, is now being used in numerous countries in the world including the United States. Following this approach, the emphasis will be put on principles rather than techniques. The "solving" of certain games can motivate children as well. The interesting thing is not only determining the one who has the best chance of winning, but for instance deciding how the winnings should be distributed if the game ends at a given point, or how long the game lasts, or whether it is possible that the game will never end. These questions will be answered simply and elegantly with the methods discussed below.

The use of calculators and PCs is gaining greater and greater importance in education today. Think of, for instance, statistics or the calculation of different means. We should not be scared to use them. Lots of schools are now equipped with so-called 'digital' blackboards which combine the standard blackboard with the most modern tools. Special attention is laid on the following: with the help of some mathematical program (MATHEmAticA, MAPLE) a quick and easy solution of a calculation can be given, which could not be solved otherwise owing to the lack of necessary methods or time. (The 5.0 version of MATHEMATicA is used in the paper. The commands do not need the previous downloading of environments.)

According to the principles of project-oriented mathematical education, which began in the US, the learning process is an active one and it can only be successful and effective if students actively participate. Hence students in pairs or groups — depending on their number — should play such games a couple of times before discussing them in class. This way students can see the point of learning and their goals. Preliminary evaluations can also be of help during calculations or a modelling task. A game could also be given as a homework for a lesson, but it should kindle a student's interest and help maintain their love of mathematics and mathematical problem-solving.

The author would like to thank József Kosztolányi for his help with the draughting of the article and Barbara Fülöp and David Curley for translating it.

\section{Tossing up to the first 'six'}

We shall begin with the most frequently played box-and-dice games. First, let us consider how players decide who should start in a board game.
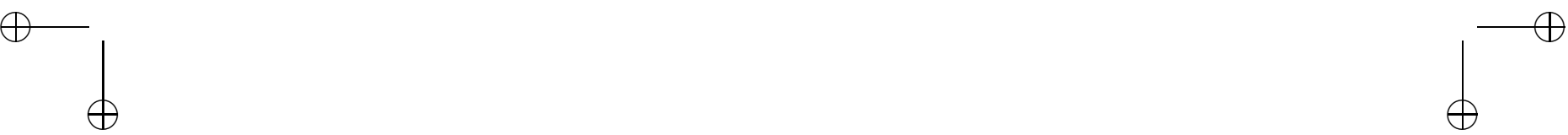


$$
\text { "mader" — 2008/7/31 — 11:38 — page } 17 \text { — \#3 }
$$

Problem 1. Two players, Anne and Bob ( $A$ and $B$ from now on) play with a regular dice. $A$ starts the game, they toss one after the other, and the winner is the one who throws a six for the first time. What is the probability of $A$ winning?

Solution (with series). Let $A$ stand for the event when $A$ wins. We want to find the value of $P(A)$. Let $P\left(A_{n}\right)$ mean that $A$ wins in the $n^{\text {th }}\left(n \in \mathbb{N}^{+}\right)$step. In this case, $P(A)=\sum_{n=1}^{\infty} P\left(A_{n}\right)$. It is easy to see that $n$ can only be an odd number, since $A$ can only win with an odd toss. Let $n=2 k+1$, where $k \in \mathbb{N}$. Then $P\left(A_{1}\right)=\frac{1}{6}$, and $P\left(A_{3}\right)=\frac{1}{6}\left(\frac{5}{6}\right)^{2}$, since for $A$ to win for the third throw, $A$ must not toss a six for the first time, and $B$ must not toss a six for the second time either, and $A$ must toss a six for the third time. A similar train of thought will lead us to the result $P\left(A_{n}\right)=\frac{1}{6}\left(\frac{5}{6}\right)^{n-1}$. The sum we have been looking for has $a_{1}=\frac{1}{6}$ as the first term, and it is the sum of an infinite series where $q=\left(\frac{5}{6}\right)^{2}$. So

$$
\sum_{n=1}^{\infty} P\left(A_{n}\right)=\sum_{k=0}^{\infty} P\left(A_{2 k+1}\right)=\sum_{k=0}^{\infty} \frac{1}{6}\left(\frac{5}{6}\right)^{2 k}=\frac{1}{6} \cdot \frac{1}{1-\frac{25}{36}}=\frac{6}{11}
$$

Remark. A question may arise; can the game last forever? The answer to this question can be found by calculating the probability of Bob winning. With the above line of thinking, we get $P(B)=\frac{5}{11}$. Then we get $P(A)+P(B)=1$, so the probability of having a never-ending game is 0 . It does not mean that the game will terminate in a finite number of steps, it just means that the probability that it lasts forever is 0 .

An event was found above whose probability is 0 , but whose occurrence is not impossible. This should mentioned at this point to put things into perspective. It can also be compared to what students are already familiar with from the viewpoint of geometric probability; the probability of hitting a certain point in a circular target is 0 , but it is not an impossible event.

Solution (with a flowsheet). The exercise can also be adapted to students who are unfamiliar with the concept of an infinite geometric series. (Being able to calculate limits is required for this, which is not part of the standard curriculum requirements). Now consider the following flowsheet (Figure 1).

$\mathrm{S}$ tells us the states at the start when $A$ tosses next; $A$ can toss a six with the probability of $\frac{1}{6}$, and then we arrive at the state where ' $A$ won'. If $A$ does not toss a six with the probability of $\frac{5}{6}$, we arrive at the $C_{1}$ state, from where $B$ tosses next. If $B$ tosses a six, he wins; the probability of this event is $\frac{1}{6}$ in this case as well, and if he does not toss a six, $A$ flips again, so we arrive at the 


$$
\text { "mader" — 2008/7/31 — 11:38 — page } 18 \text { — \#4 }
$$

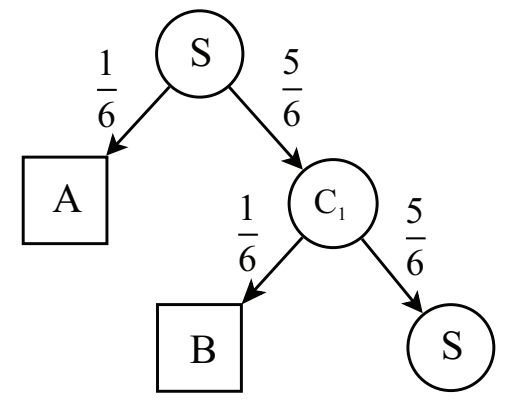

Figure 1. Tossing for the first 'six'

original state $S$. (We continue the game from the state $S$, and do not show again the states that occurred previously.) We now want to know the probability of $A$ winning with the $S$ state. Let us represent this by $P_{S}(A)$, and the other states in a similar way. Then we get the following set of equations:

$$
\begin{aligned}
P_{S}(A) & =\frac{1}{6}+\frac{5}{6} P_{C_{1}}(A) \\
P_{C_{1}}(A) & =\frac{5}{6} P_{S}(A)
\end{aligned}
$$

From this we have $P_{S}(A)=\frac{6}{11}$. When solving this set of equations we find that $P_{S}(A)=\frac{\frac{1}{6}}{1-\left(\frac{5}{6}\right)^{2}}$ which is exactly the same value as that for the classical empirical formula of the above-mentioned geometric series. We also get $P_{C_{1}}(A)=\frac{5}{11}$. From the state $C_{1} B$ tosses, so $P_{C_{1}}(A)=P(B)$. (The same method can also be used to calculate the probability of $B$ winning as well.)

In the above the calculation with an infinite series was reduced to the solving of a linear set of equations. This trick makes it possible for us to present and do the problem in secondary schools as well. The probability of $A$ winning from any of the conditions could also be given with the same set of equations. For this result we should have used the sum of the terms of another series.

Remark. The fact that $A$ has an advantage is obvious even before the commencement of the game, since $B$ can only win if $A$ does not toss a six for the first time, and the probability of this event is not 1 . The game therefore is not fair. (The question of whether it could be made fair will be discussed later on.)

The asymmetrical nature of the game is better seen if another version of the flowsheet above is drawn (Figure 2). 


$$
\text { "mader" — 2008/7/31 — 11:38 — page } 19-\# 5
$$

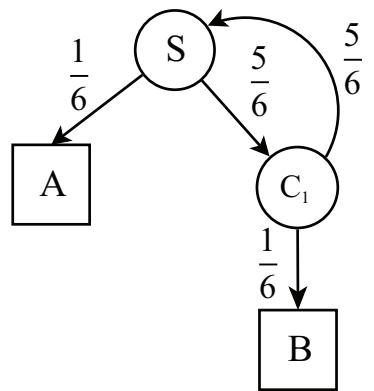

Figure 2. Tossing for the first 'six'

With this type of flowsheet, we do not show any of the states that have occurred previously, so the flow of the game can be viewed as a walk in a directed graph.

Remark. There is no great difference between the two flowsheets. While the structure of the game is clearer in the first one, the train of thought can be better followed in the second one. The latter has a disadvantage though; it becomes complicated if the number of states is increased. It is also recommended that you start the introduction of the game with the first flowsheet in the classroom. (Actually, it is a finite representation of an infinite regular tree.)

A flowsheet diagram for a similar game can be easily drawn based on the above diagram. The flowsheet usually contains the states with a different notation for the final states. An arrow is directed from each state to those states that can be reached from there with a positive probability. The probability is always indicated on the arrow. In addition to this, the given event is often indicated as well.

Those processes where there is a finite set of states and the probability of arriving at a given state can be expressed by the product of the previous state's probability and the transition probability are called Markov chains.

When teaching probability theory in secondary schools, we think that these flowsheets are ideally suited for representing Markov chains.

A similar flowsheet can be drawn if we are only interested in finding the probability of one person winning, and not in the flow of the game (see Figure 3).

It is important to emphasise that instead of representing the states, we can calculate the probability of one player winning in a given state. The flowsheet 


$$
\text { "mader" — 2008/7/31 — 11:38 — page } 20-\# 6
$$

below is better than the previous one, because the probability of $A$ winning can be found in a straightforward way with the help of a linear equation.

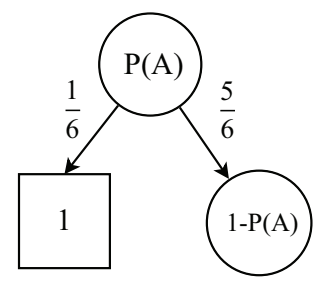

Figure 3. Tossing for the first 'six'

$$
P(A)=\frac{1}{6}+\frac{5}{6}(1-P(A))
$$

The probability that a game like this will last forever is 0 , as we have already seen. So in how many steps will it probably end? Figure 1(2) can give us the answer to this question as well.

Problem 2. Find the expected value of the number of throws in the abovementioned game.

Solution (with series). The expected value can be found with the help of the following infinite sum:

$$
1 \cdot \frac{1}{6}+2 \cdot \frac{5}{6} \cdot \frac{1}{6}+3 \cdot \frac{5}{6} \cdot \frac{5}{6} \cdot \frac{1}{6}+\ldots=\sum_{n=1}^{\infty} \frac{n \cdot 5^{n-1}}{6^{n}}
$$

This means that the game might end with the first toss (probability $\frac{1}{6}$ ) or with the second toss (probability $\frac{5}{6} \cdot \frac{1}{6}$ ), and so on. Since the calculation of such expressions is beyond the limits of secondary mathematics education, even at the upper level, using a mathematical program like MATHEMATICA might be a good idea. The sum can be computed by entering the following command:

$\operatorname{Sum}\left[\left(n * 5^{\wedge}(n-1)\right) / 6^{\wedge} n,\{n, 1\right.$, Infinity $\left.\}\right]$

We can also sum the above series on paper if the theory of infinite geometric series can be used. Let us modify the above-mentioned sum in the following way: $\sum_{n=1}^{\infty} \frac{n \cdot 5^{n-1}}{6^{n}}=\frac{1}{5} \sum_{n=1}^{\infty} n \cdot\left(\frac{5}{6}\right)^{n}$. This sum can be calculated as the sum of the 


$$
\text { "mader" — 2008/7/31 - 11:38 — page } 21 \text { — \#7 }
$$

sum of the following cleverly written, infinitely many geometric series:

$$
\begin{aligned}
\frac{5}{6}+\left(\frac{5}{6}\right)^{2}+ & \left(\frac{5}{6}\right)^{3}+\left(\frac{5}{6}\right)^{4}+\ldots \\
\left(\frac{5}{6}\right)^{2}+ & \left(\frac{5}{6}\right)^{3}+\left(\frac{5}{6}\right)^{4}+\ldots \\
& \left(\frac{5}{6}\right)^{3}+\left(\frac{5}{6}\right)^{4}+\ldots \\
& \left(\frac{5}{6}\right)^{4}+\ldots
\end{aligned}
$$

Adding up the sum of above geometric series leads to the following geometric series

$$
\frac{\frac{5}{6}}{1-\frac{5}{6}}+\frac{\left(\frac{5}{6}\right)^{2}}{1-\frac{5}{6}}+\frac{\left(\frac{5}{6}\right)^{3}}{1-\frac{5}{6}}+\ldots,
$$

which can be written in the following simple way:

$$
5+5 \cdot \frac{5}{6}+5 \cdot\left(\frac{5}{6}\right)^{2}+\ldots=30
$$

The expected value we are looking for is equal to $1 / 5$ of this, which is 6 . We used the fact that the series is absolutely convergent, so the sum has a finite value, and for each permutation the final sum is the same.

Remark. It is a good idea to ask students to estimate the expected value before doing the calculation. It is easy to estimate this if we realize that we have to keep flipping until we get the first six. The result should not surprise us if we realize that the required number of tosses up to the first six is a random variable of a geometric distribution where the parametric variable is $p=\frac{1}{6}$ and the expected value is $\frac{1}{p}=6$.

Solution (with a flowsheet). We can find the solution using elementary methods and with the help of Figure 1(2). Let $l_{S}$ and $l_{C_{1}}$ stand for the expected values of the number of steps from states $\mathrm{S}$ and $C_{1}$. Then using the flowsheet method we get the following set of equations:

$$
\begin{aligned}
l_{S} & =\frac{1}{6}+\frac{5}{6}\left(1+l_{C_{1}}\right) \\
l_{C_{1}} & =\frac{1}{6}+\frac{5}{6}\left(1+l_{S}\right)
\end{aligned}
$$


This means that the game either ends with the probability of $\frac{1}{6}$ after one throw, or we arrive at state $C_{1}$ with a probability of $\frac{5}{6}$, after which the $l_{C_{1}}$ step is needed.

The solution of the system of equations is $l_{S}=l_{C_{1}}=6$. The solution can also be found by using the following command (we rename the variables $l_{S}=a$, $\left.l_{C_{1}}=b\right)$ :

Solve $[\{\mathrm{a}==1 / 6+5 / 6(1+\mathrm{b}), \mathrm{b}==1 / 6+5 / 6(1+\mathrm{a})\},\{\mathrm{a}, \mathrm{b}\}]$

The program will be used later on, when more complex systems of equations need to be solved.

Remark. The calculations become more difficult with series, some being almost insoluble using the current methods taught in secondary schools. However, with flowsheet diagrams like those shown above many problems can be reduced to solving a linear set of simultaneous equations, which is taught in the school curriculum.

The above-mentioned problem can be generalized in many ways, like the one given next.

Problem 3. Three players - $A, B$ and $C-$ play with a regular dice. $A$ starts the game, they toss one after the other. The winner is the one who tosses a six for the first time. What is the probability of $A$ winning?

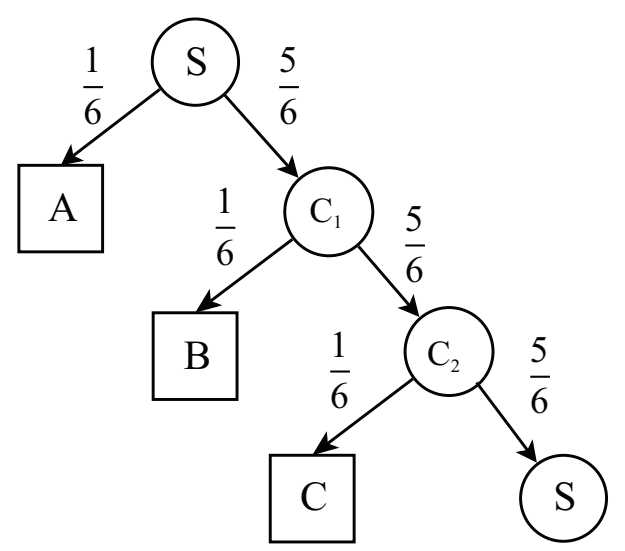

Figure 4. Tossing until the first 'six' 


$$
\text { "mader" — 2008/7/31 - 11:38 — page } 23 \text { — \#9 }
$$

Solution. Following the previous train of thought, either working with a series or using a flowsheet, the answer can be readily found. In the case of a series, the probability estimate we are looking for is given by an infinite geometric series with $a_{1}=\frac{1}{6}$ as the initial term, and $q=\left(\frac{5}{6}\right)^{3}$ as the quotient. With this we get $P(A)=\frac{36}{91}$.

Based on a flowsheet (see Figure 4), a set of equations can be written down, from which the probability of $A, B$ or $C$ winning can be easily calculated.

Going to the general case, we have $n$ players, and we would like to know the probability of the $k^{\text {th }}$ player winning. The solution is $p=\frac{1}{6}\left(\frac{5}{6}\right)^{k-1} \frac{1}{1-\left(\frac{5}{6}\right)^{n}}$.

\section{Turning a flowsheet problem into a game task}

The whole thing can also be turned around. It would be fun, but it is a lot more difficult to find a game task for a flowsheet that describes it. However, in principle, given a game task one can find the corresponding flowsheet problem, or vice versa.

Problem 4. Given the following flowsheet, what game task does it correspond to (Figure 5)?

Solution. For example, two players roll a dice, each in turn. The winner is the one who tosses a number that has already been rolled during the game.

Remark. It can be easily seen, that the players can't reach any previousreached state, so the game can not be never-ending. Hence a simple calculation is adequate, namely: $P(A)=\frac{5}{6} \cdot \frac{2}{6}+\frac{5}{6} \cdot \frac{4}{6} \cdot \frac{3}{6} \cdot \frac{4}{6}+\frac{5}{6} \cdot \frac{4}{6} \cdot \frac{3}{6} \cdot \frac{2}{6} \cdot \frac{1}{6}=\frac{620}{1296}$ and $P(B)=1-P(A)=\frac{676}{1296}$. (The probability of either player winning can also be calculated by solving a set of equations.) The game can easily be generalized to one involving three or more players, which gives rise to other problems and questions as well. How many people can play? If we could choose when to toss, when should we do it? These questions are actually easy to answer. The number of players cannot exceed seven, since if no one wins after the first six throws, it means that all six players tossed different numbers, so the seventh player would necessarily toss a number that has already come up. In the case of seven players if $P\left(A_{i}\right)$ stands for the probability of the $i^{\text {th }}$ player winning then $P\left(A_{1}\right)=0$, $P\left(A_{2}\right)=\frac{1}{6}, P\left(A_{3}\right)=\frac{5}{6} \cdot \frac{2}{6}, P\left(A_{4}\right)=\frac{5}{6} \cdot \frac{4}{6} \cdot \frac{3}{6}, P\left(A_{5}\right)=\frac{5}{6} \cdot \frac{4}{6} \cdot \frac{3}{6} \cdot \frac{4}{6}, P\left(A_{6}\right)=\frac{5}{6} \cdot \frac{4}{6} \cdot \frac{3}{6} \cdot \frac{2}{6} \cdot \frac{5}{6}$, 


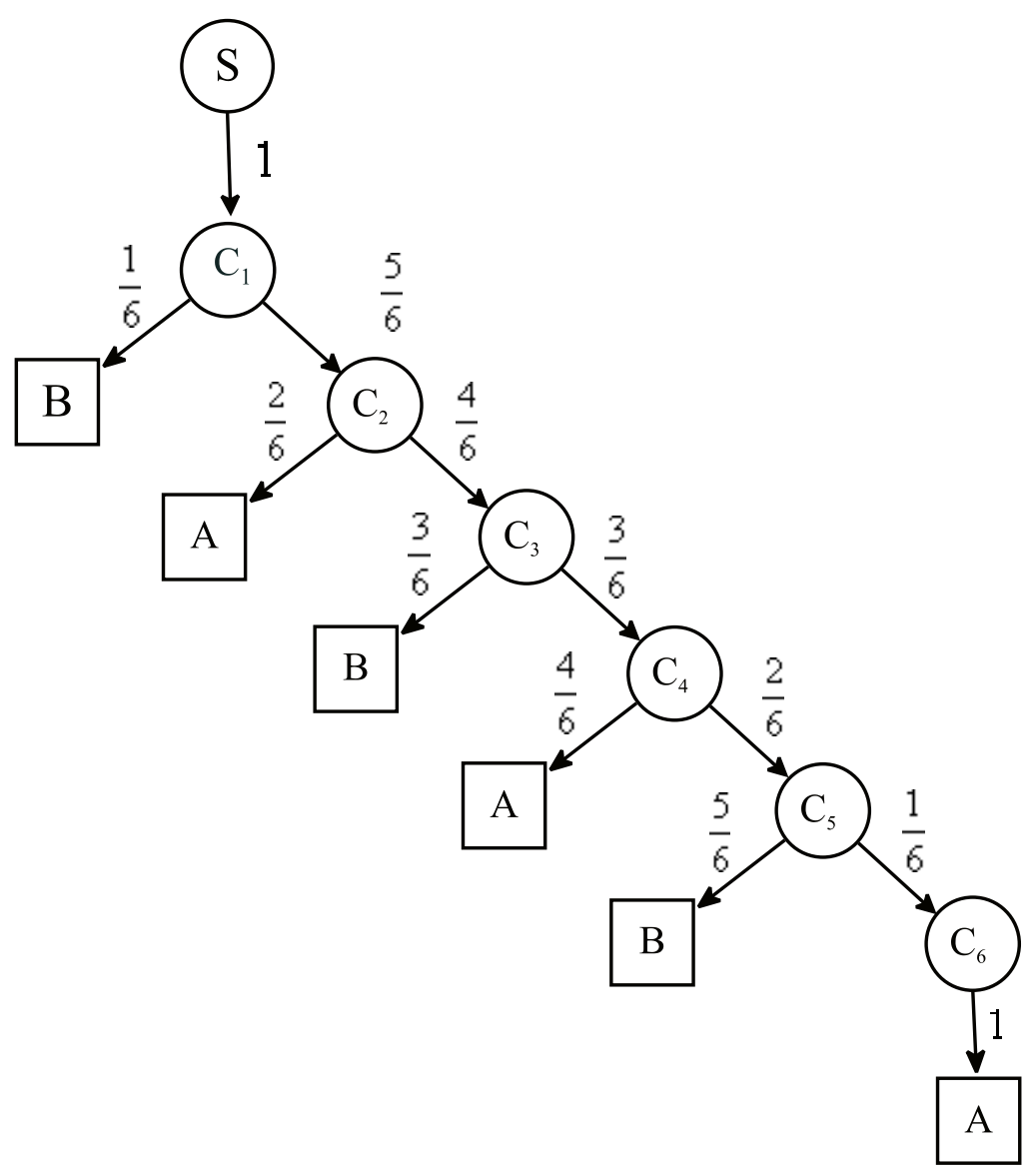

Figure 5. What is the game?

$P\left(A_{7}\right)=\frac{5}{6} \cdot \frac{4}{6} \cdot \frac{3}{6} \cdot \frac{2}{6} \cdot \frac{1}{6} \cdot 1$. Obviously the third and fourth players have the best chance of winning, and this probability is $P\left(A_{3}\right)=P\left(A_{4}\right)=\frac{10}{36}$. The flowsheet displayed above can be easily changed to $n(n=2,3, \ldots, 7)$ players, but only the players' names should be changed. The above calculation can also be used for fewer than seven players.

The flowsheets for the two game tasks, and solutions got from methods that do not use flowsheets must be related. And the common core of each problem is quite evident; it does not matter whether we toss a dice or pull out slips of paper 


$$
\text { "mader" — 2008/7/31 — 11:38 — page } 25 \text { — \#11 }
$$

that contain the numbers $1,2,3,4,5$ or 6 with replacement. Coin tossing can also be easily substituted by changing it to drawing with replacement.

Another generalization of the above-mentioned tasks can be derived from this:

\section{Sampling with replacement}

Problem 5. Let us put $n$ balls - a white and b black balls - into an urn. Two players - $A$ and $B$ - pull a ball out one after the other and replace them. The winner is the one who pulls out a white ball for the first time. What is the probability of $A$ winning?

Solution. Let $p=\frac{a}{n}$. In this case $1-p=\frac{b}{n}$.

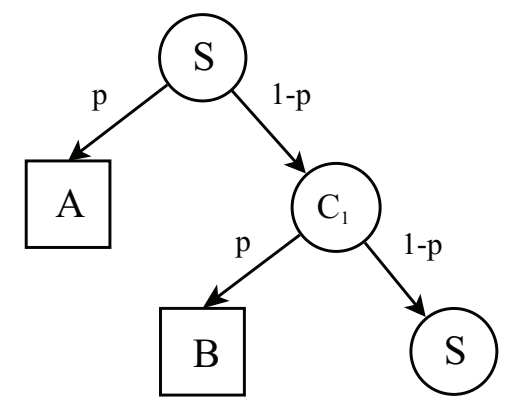

Figure 6. Sampling with replacement

Based on the following flowsheet we can get the probability values by solving the following set of equations:

$$
\begin{aligned}
P_{S}(A) & =p+(1-p) P_{C_{1}}(A) \\
P_{C_{1}}(A) & =(1-p) P_{S}(A)
\end{aligned}
$$

solving the system of equations

$$
\begin{aligned}
P_{S}(A) & =p+(1-p)^{2} P_{S}(A) \\
\left(1-(1-p)^{2}\right) P_{S}(A) & =p \\
P_{S}(A) & =\frac{p}{1-(1-p)^{2}} .
\end{aligned}
$$




$$
\text { "mader" — 2008/7/31 — 11:38 — page } 26 \text { — \#12 }
$$

We have already seen in Problem 1 that the first player has an advantage, because he has the best chance of winning. Therefore the problem of making it fair arises in connection with its generalization.

Problem 6. Can such a game be made fair by the biasing of the dice, or the coin, and by changing the number, and the ratio of balls in different colours appropriately?

Solution. The above-mentioned method with replacement can also be understood by having a situation where we play with a biased coin and the probability of tossing a head is $p$, where $0<p<1$, and the probability of tossing a tail is $1-p$. The game is fair if $P_{S}(A)=P_{S}(B)=\frac{1}{2}$, so we are looking for the value of $p$ for which $P_{S}(A)=\frac{p}{1-(1-p)^{2}}=\frac{1}{2}$. However $P_{S}(A)=\frac{p}{1-(1-p)^{2}}=\frac{1}{2-p} \neq \frac{1}{2}$, if $0<p<1$. The game cannot be made fair. The first player will always have an advantage.

A not so different problem is worth mentioning at this point.

Problem 7. Is it possible to draw lots with a biased coin fairly?

Solution. Let the probability of tossing a head be $p$ and the probability of tossing a tail be $1-p$ where $0<p<1$. Let us toss the coin twice and let $A$ win if the result is th, (tails, heads) and $B$ if it is ht, (heads, tails) otherwise the tossing should be repeated. The game is fair, since the probability of both sequences is $p(1-p) \neq 0$. It does not matter who tossed the given sequence; only the result of the toss counts.

Another popular procedure in combinatorics is sampling without replacement. These types of problems in probability theory can also be solved with the help of flowsheets.

\section{Sampling without replacement}

Problem 8. An urn contains two red and three blue balls. Pull out one ball at a time without replacement. Stop the process if both a red and a blue ball are among the balls that were pulled out. What is the probability of having exactly $n,(n=1,2, \ldots, 5)$ draws? 


$$
\text { "mader" — 2008/7/31 — 11:38 — page } 27 \text { — \#13 }
$$

Solution. Look at the following flowsheet (Figure 7).

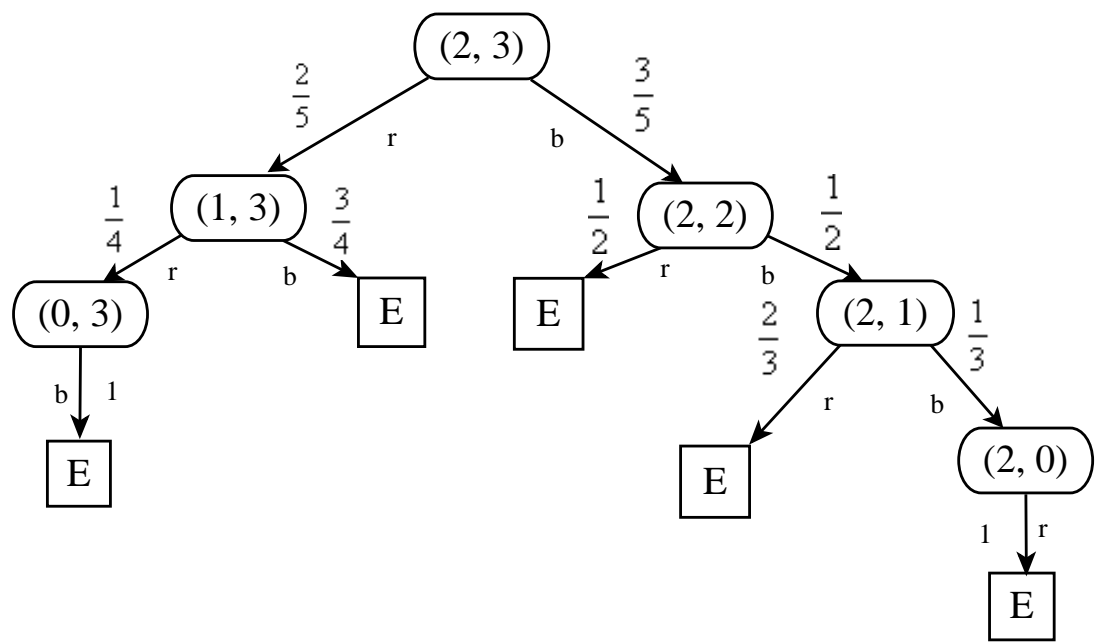

Figure \%. Sampling without replacement

In this case $(r, b)$ means that there are $r$ red and $b$ blue balls in the urn. $E$ means the end of the game, when one red and one blue ball have been pulled out. The colour of the ball pulled out, and its probable value are indicated on the arrows. The probability values that we are looking for can be easily read from the flowsheet. Let $p_{n}$ be the probability of having both a blue and a red ball pulled out for the $n^{\text {th }}$ draw. Then $p_{1}=0, p_{2}=\frac{2}{5} \cdot \frac{3}{4}+\frac{3}{5} \cdot \frac{1}{2}=\frac{6}{10}, p_{3}=\frac{2}{5} \cdot \frac{1}{4} \cdot 1+\frac{3}{5} \cdot \frac{1}{2} \cdot \frac{2}{3}=\frac{3}{10}$, $p_{4}=\frac{3}{5} \cdot \frac{1}{2} \cdot \frac{1}{3}=\frac{1}{10}, p_{5}=0$. We can see, that the game cannot be neverending, because the players can not reach any formerly-reached state, just like in Problem 4.

The flowsheet method can assist us in the case of more difficult problems as well.

Problem 9. There are five black and three white balls in an urn. One is pulled out and three are put back into the urn with the same colour. Another one is pulled out again. What is the probability of pulling out two balls with the same colour and two with different colours?

Solution. Noting the previous exercise, we can draw a simple flowsheet for this problem as well. In this case $(w, b)$ means that there are $w$ white and $b$ black 
balls in the urn. Beside the probability values, the colour of the balls pulled out is indicated on the arrows (Figure 8).

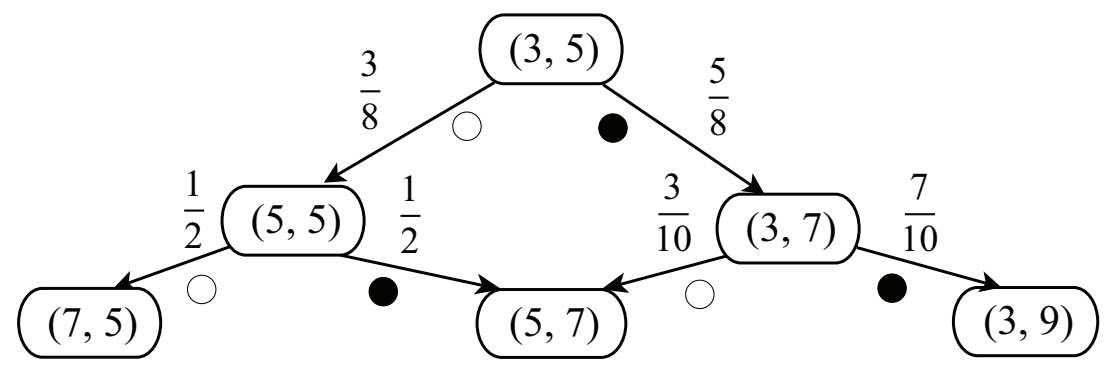

Figure 8. Sampling without replacement

Let $A$ be the event when two balls are pulled with the same colour, and $B$ the event when the balls pulled out have different colours. In this case, using the flowsheet, $P(A)=\frac{3}{8} \cdot \frac{1}{2}+\frac{5}{8} \cdot \frac{7}{10}=\frac{5}{8}$, and $P(B)=\frac{3}{8} \cdot \frac{1}{2}+\frac{5}{8} \cdot \frac{3}{10}=\frac{3}{8}$.

Remark. Conditional probability can also be introduced by this method: If the first draw yields a black ball, what is the probability it being a white ball the next time?

Looking at the figure, it can readily be seen that the probability is $p=\frac{3}{10}$. It is also clear how the event space is altered after the first draw.

Conditional probability and the concept of independent events are hard for secondary students, but they can be easily discussed with this graphical method.

Sometimes a 'simply worded' combinatorical problem results in a counting task that is almost impossible for students to do. With the help of a flowsheet, however, this type of problem should be no more difficult than the ones we have tackled so far.

\section{A heads or tails game - heads after tails}

A dice is something that not many people carry in the pocket, but a coin is something that most people have.

Problem 10. $A$ and $B$ toss a regular coin one after the other. The winner is the one who tosses a head after a tail for the first time. What is the probability of $A$ winning if $A$ starts the game? 


$$
\text { "mader" — 2008/7/31 — 11:38 — page } 29 \text { — \#15 }
$$

The problem can be solved with the help of a flowsheet or by using combinatorics theory. We shall take a look at the latter case, then the former case.

Solution (with series). Let us suppose that $A$ wins in the $k^{\text {th }}$ step. The number of "heads-and-tails" series with length $k$ is $2^{k}$, and the probability of their occurrence is $\frac{1}{2^{k}}$ for each case. The good events are "heads-and-tails" series with length $k$, where a head comes last and if there is another head in the series it must follow that one as well since a th sequence can only appear at the end. The good events are given by the following "heads-and-tails" series with length $k$

$$
\begin{gathered}
\text { ttt...tth } \\
\text { htt...tth } \\
\text { hht...tth } \\
\vdots \\
\text { hhh...hth }
\end{gathered}
$$

Their number is $k-1$. So $P\left(A_{k}\right)=\frac{k-1}{2^{k}}$, taking into consideration the fact that $A$ can only win for each odd throw. Using the notation $k=2 n+1, n \in \mathbb{N}$ :

$$
P(A)=\sum_{k \text { odd }} \frac{k-1}{2^{k}}=\sum_{n=1}^{\infty} \frac{2 n}{2^{2 n+1}}=\sum_{n=1}^{\infty} \frac{n}{4^{n}} .
$$

Since this series is not taught in the school curriculum, we will apply the approach that we used earlier, namely,

$$
\begin{aligned}
& \frac{1}{4}+\left(\frac{1}{4}\right)^{2}+\left(\frac{1}{4}\right)^{3}+\left(\frac{1}{4}\right)^{4}+\ldots \\
&\left(\frac{1}{4}\right)^{2}+\left(\frac{1}{4}\right)^{3}+\left(\frac{1}{4}\right)^{4}+\ldots \\
&\left(\frac{1}{4}\right)^{3}+\left(\frac{1}{4}\right)^{4}+\ldots \\
&\left(\frac{1}{4}\right)^{4}+\ldots
\end{aligned}
$$

The sum of these series once again yields an infinite geometric series that has the form

$$
\frac{\frac{1}{4}}{1-\frac{1}{4}}+\frac{\left(\frac{1}{4}\right)^{2}}{1-\frac{1}{4}}+\frac{\left(\frac{1}{4}\right)^{3}}{1-\frac{1}{4}}+\ldots
$$




$$
\text { "mader" — 2008/7/31 — 11:38 — page } 30 \text { — \#16 }
$$

which can be written in the following way:

$$
\frac{1}{3}+\frac{1}{3} \cdot \frac{1}{4}+\frac{1}{3} \cdot\left(\frac{1}{4}\right)^{2}+\ldots=\frac{4}{9}
$$

So we get $P(A)=\frac{4}{9}$.

Solution (with the help of a flowsheet). A simpler solution can be given for this. First, draw the graph of the game (see Figure 9). The starting state is indicated by an $S$, and the probability of tossing a head or tail is $\frac{1}{2}-\frac{1}{2}$ for each case thereafter. We can arrive at states $C_{1}$ and $C_{2}$ from $S$, where $B$ tosses next. If $B$ flips a tail in the $C_{1}$ state, he wins. If $B$ tosses a head, $A$ comes next. A tail was tossed before, so we arrive at state $C_{3}$. If $B$ tosses a head in the $C_{2}$ state, $A$ comes next. Neither has tossed a tail before so we get back the the original $S$ state. However $B$ tosses a head in $C_{2}, A$ flips and gets a tail like before, so we arrive at the $C_{1}$ state again. $A$ wins if a head is tossed in the $C_{3}$ state, otherwise we arrive at the $C_{1}$ state again.

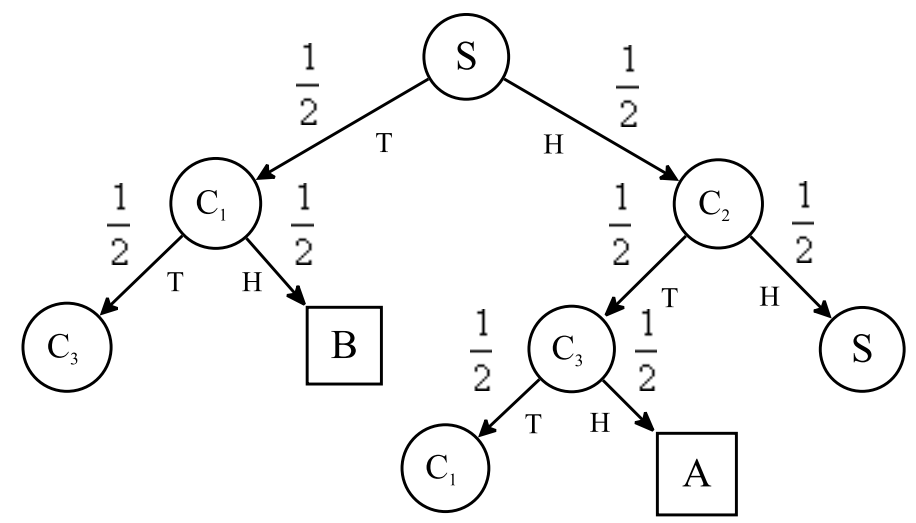

Figure 9. One head after a tail

Using the usual notation, the system of equations is the following:

$$
\begin{aligned}
P_{S}(A) & =\frac{1}{2} P_{C_{1}}(A)+\frac{1}{2} P_{C_{2}}(A) \\
P_{C_{1}}(A) & =\frac{1}{2} P_{C_{3}}(A) \\
P_{C_{2}}(A) & =\frac{1}{2} P_{C_{3}}(A)+\frac{1}{2} P_{S}(A)
\end{aligned}
$$




$$
\text { "mader" — 2008/7/31 — 11:38 — page } 31 \text { — \#17 }
$$

$$
P_{C_{3}}(A)=\frac{1}{2} P_{C_{1}}(A)+\frac{1}{2}
$$

Solving this set of equations, we get $P_{S}(A)=\frac{4}{9}$. Calculating the probability of $B$ winning we get $P(B)=\frac{5}{9}$, so the probability of a never-ending game is 0 in this case as well. $B$ has an advantage since he has the chance of winning first on the second toss.

Remark. Another flowsheet can be drawn to illustrate the structure of the game and its asymmetric nature. This is what it looks like (Figure 10):

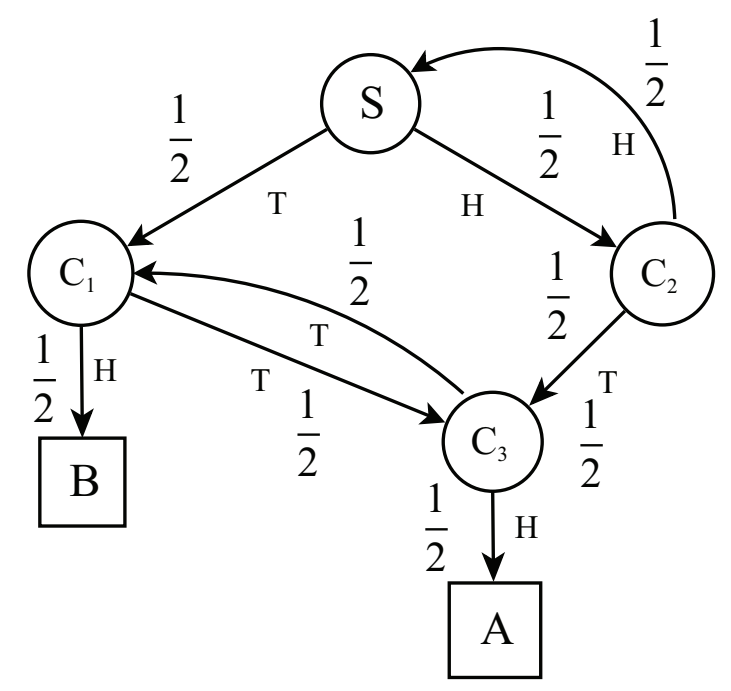

Figure 10. One head after a tail

7. A fair heads or tails game

Problem 11. $A$ and $B$ flip a regular coin one after the other. The winner is the one who tosses the $n^{\text {th }}$ head. What is the chance of $A$ winning if $A$ starts the game?

Solution (with recursion). At the start of the game the probability of $A$ winning is $P\left(A_{n}\right)$. A tosses a head or tail with a probability of $\frac{1}{2}-\frac{1}{2}$, after which 
the winning probability is $P\left(A_{n}\right)$ or $P\left(A_{n-1}\right)$ for the next player (who is $B$ ). So the winning probability for $A$ is $1-P\left(A_{n}\right)$ or $1-P\left(A_{n-1}\right)$ before the second hit.

(Figure 11)

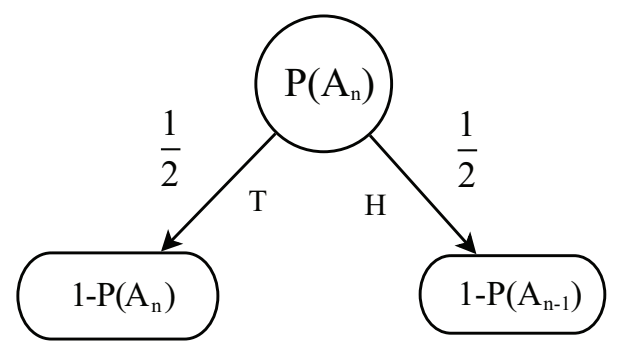

Figure 11. Tossing the $n^{\text {th }}$ head

In this case for $P\left(A_{n}\right)$, we get the following recursion relation

$$
P\left(A_{n}\right)=\frac{1}{2} \cdot\left(1-P\left(A_{n-1}\right)\right)+\frac{1}{2} \cdot\left(1-P\left(A_{n}\right)\right)
$$

Hence we see that

$$
P\left(A_{n}\right)=\frac{2-P\left(A_{n-1}\right)}{3} .
$$

Since $P\left(A_{1}\right)=\frac{2}{3}$ is already known, the first few $P\left(A_{n}\right)$ values can be calculated. $P\left(A_{1}\right)=\frac{2}{3}, P\left(A_{2}\right)=\frac{4}{9}, P\left(A_{3}\right)=\frac{14}{27}$, and so on. This leads us to suppose that for the $n^{\text {th }}$ case

$$
P\left(A_{n}\right)=\frac{1}{2} \cdot\left(1+\frac{(-1)^{n+1}}{3^{n}}\right) .
$$

The proof can be given by induction.

That the formula is correct for the $n=1,2,3$, can be shown by putting the values in. As for the inductive hypothesis, let us suppose that the statement is true for any case up to $n$. Our aim is to show that it is also true for $n+1$ as well, so we need to prove that $P\left(A_{n+1}\right)=\frac{1}{2}+\frac{1}{2} \cdot \frac{(-1)^{n+2}}{3^{n+1}}$. In this case $3 P\left(A_{n+1}\right)=2-P\left(A_{n}\right)=2-\frac{1}{2}-\frac{1}{2} \cdot \frac{(-1)^{n+1}}{3^{n}}$, so

$$
P\left(A_{n+1}\right)=\frac{1}{2}-\frac{1}{2} \cdot \frac{(-1)^{n+1}}{3^{n+1}}=\frac{1}{2}+\frac{1}{2} \cdot \frac{(-1)^{n+2}}{3^{n+1}}
$$

which is just what we intended to show.

We can also derive the explicit form using the notation, $P\left(A_{n}\right)=a_{n}$. Here, then, we would like to give an explicit form for the $n^{\text {th }}$ term of the linear recursion 


$$
\text { "mader" — 2008/7/31 — 11:38 — page } 33 \text { — \#19 }
$$

formula, where $a_{1}=\frac{2}{3}$ and $a_{n}=-\frac{1}{3} a_{n-1}+\frac{2}{3}$. Writing the first couple of terms

$$
\begin{aligned}
& a_{1}=\frac{2}{3} \\
& a_{2}=-\frac{1}{3} a_{1}+\frac{2}{3} \\
& a_{3}=-\frac{1}{3} a_{2}+\frac{2}{3} \\
& a_{4}=-\frac{1}{3} a_{3}+\frac{2}{3} \\
& a_{5}=-\frac{1}{3} a_{4}+\frac{2}{3}
\end{aligned}
$$

Now let us take a look at the $d_{i}=a_{i+1}-a_{i}$ series of differences:

$$
\begin{aligned}
& d_{1}=a_{2}-a_{1}=-\frac{1}{3} a_{1} \\
& d_{2}=a_{3}-a_{2}=-\frac{1}{3}\left(a_{2}-a_{1}\right)=-\frac{1}{3} d_{1} \\
& d_{3}=a_{4}-a_{3}=-\frac{1}{3}\left(a_{3}-a_{2}\right)=-\frac{1}{3} d_{2} \\
& d_{4}=a_{5}-a_{4}=-\frac{1}{3}\left(a_{4}-a_{3}\right)=-\frac{1}{3} d_{3}
\end{aligned}
$$

Proceeding this way, we arrive at the $d_{n}$ case with $d_{1}=-\frac{1}{3} a_{1}$ as the first term and $q=-\frac{1}{3}$ as the common multiplicative factor of the geometric series. Here the $a_{n}=d_{n-1}+a_{n-1}$ pattern can clearly be seen. Rewriting the equations like so

$$
\begin{aligned}
a_{n} & =d_{n-1}+a_{n-1} \\
a_{n-1} & =d_{n-2}+a_{n-2} \\
& \vdots \\
a_{2} & =d_{1}+a_{1}
\end{aligned}
$$

we get

$$
a_{2}+a_{3}+\ldots+a_{n}=d_{1}+d_{2}+\ldots+d_{n-1}+a_{1}+a_{2}+\ldots+a_{n-1} .
$$


After arranging and substituting we find that

$$
\begin{aligned}
a_{n} & =\frac{2}{3}+\frac{\left(-\frac{1}{3}\right)^{n-1}-1}{\left(-\frac{1}{3}\right)-1} \cdot\left(-\frac{1}{3}\right) \frac{2}{3}=\frac{2}{3}+\frac{\left(-\frac{1}{3}\right)^{n-1}-1}{2} \cdot \frac{1}{3} \\
& =\frac{4+\left(-\frac{1}{3}\right)^{n-1}-1}{6}=\frac{1}{2}+\frac{1}{2} \cdot \frac{-\left(\frac{1}{3}\right)^{n-1}}{3} \\
& =\frac{1}{2} \cdot\left(1+\frac{(-1)^{n-1}}{3^{n}}\right)=\frac{1}{2} \cdot\left(1+\frac{(-1)^{n+1}}{3^{n}}\right)
\end{aligned}
$$

An explicit form of the recursion relation can also be obtained using the MATHEMATiCA program after entering this command

RSolve $[\{f[n+1]==(2-f[n]) / 3, f[1]==2 / 3\}, f, n]$

It is obvious that when $n \rightarrow \infty, P\left(A_{n}\right) \rightarrow \frac{1}{2}$. In other words when $n \rightarrow \infty$, the game will be fair in mathematical terms.

Remark. In this case it is worthwhile making them draw the tree for the first couple of values of $n$ and then asking them to work out the probability values for each case.

Solution (without recursion). Let us suppose that $A$ wins on the $M^{\text {th }}$ toss, which means that the $n^{\text {th }}$ head in the game has been tossed. $M$ is odd in this case of course, so $M=2 L+1$. Our series is of length $2 L+1$, its last term being a head, and there were exactly $n-1$ heads previously. There are $\left(\begin{array}{c}2 L \\ n-1\end{array}\right)$ of this type of series with the same probability of occurrence $\left(\frac{1}{2^{2 L+1}}\right)$. The probability formula we are looking for can also be represented by the series

$$
P\left(A_{n}\right)=\sum_{L=1}^{\infty} \frac{\left(\begin{array}{c}
2 L \\
n-1
\end{array}\right)}{2^{2 L+1}}
$$

This formula yields the values $P\left(A_{1}\right)=\frac{2}{3}, P\left(A_{2}\right)=\frac{4}{9}, P\left(A_{3}\right)=\frac{14}{27}$ for $n=1,2,3$. Hence

$$
\frac{1}{2} \cdot\left(1+\frac{(-1)^{n+1}}{3^{n}}\right)=\sum_{L=1}^{\infty} \frac{\left(\begin{array}{c}
2 L \\
n-1
\end{array}\right)}{2^{2 L+1}}
$$

Remark. The solution obtained using the flowsheet was a lot easier in this case as well. Recursion relations seem to be more natural and obvious for secondary students than infinite series. Furthermore, the flowsheet model created for the problem appears to mirror the mathematical nature of the problem in a clear and straightforward way. 


$$
\text { "mader" — 2008/7/31 — 11:38 — page 35 — \#21 }
$$

The probability of the occurrence of any three-length-series is $p=\frac{1}{8}$. It is interesting, though, that these series are not equally likely.

\section{In a heads or tails game perhaps it is hhh or hth}

Problem 12. $A$ and $B$ flip a regular coin, each in turn. Only the last three tosses are observed. $A$ wins if it is hhh and $B$ wins if it is hth. Who has an advantage? It does not matter who tosses, only which series comes out first.

Solution. Based on our previous examples the flowsheet of the game can be easily drawn (Figure 12).

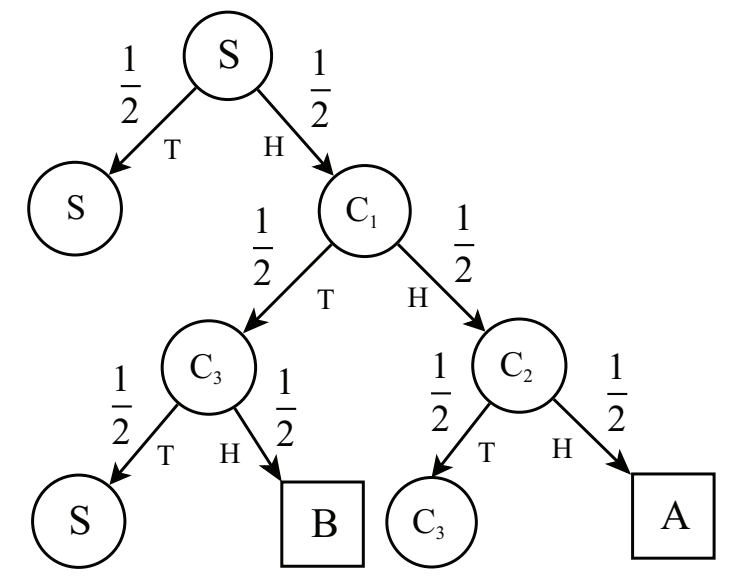

Figure 12. hhh or hth

From here with the help of the appropriate system of equations we get $P(A)=\frac{2}{5}$ and $P(B)=\frac{3}{5}$.

Remark. Similar to what we saw above, any two of the heads-and-tails series with a length of three can be played against each other. It is worth examining whether the order of the coins changes anything. Rather interestingly, it does. For example if $B$ 's good series is thh, then the probability of $A$ winning increases to $\frac{7}{8}$. But if it is hht, then the game is fair and the probability of $A$ and $B$ winning is the same $\left(\frac{1}{2}\right)$. 


$$
\text { "mader" — 2008/7/31 — 11:38 — page 36 — \#22 }
$$

A generalization of this problem will now be given.

Problem 13. Can the game be made fair with an appropriate biasing?

Solution. Let the probability of tossing a head be $p$, where $0<p<1$. Then this problem can be represented by the following system of equations:

$$
\begin{aligned}
P_{S}(A) & =(1-p) \cdot P_{S}(A)+p \cdot P_{C_{1}}(A) \\
P_{C_{1}}(A) & =(1-p) \cdot P_{C_{3}}(A)+p \cdot P_{C_{2}}(A) \\
P_{C_{2}}(A) & =(1-p) \cdot P_{C_{3}}(A)+p \\
P_{C_{3}}(A) & =(1-p) \cdot P_{S}(A)
\end{aligned}
$$

Whence we find that $P_{S}(A)=\frac{p}{1+p-p^{2}}$. The game is fair if this value is $\frac{1}{2}$. The positive root of our equation (which is quadratic in $p$ ) is $p=\frac{-1+\sqrt{5}}{2}$.

The game can be generalized without changing the players' odds of winning. It turns out that the situation does not fundamentally change if we insist on tossing an arbitrary number of heads from both players at the commencement of the game.

Problem 14. A and $B$ both flip a regular coin, each in turn. Afterwards, just the results of the last $n$ tosses are taken into consideration. $A$ wins if it is hh...hhh and $B$ wins if it is hh...hth, where we insist on tossing $n$ heads in the case of $A$ and $n-2$ heads precede the th sequence in the case of $B$. What is the probability of $A$ winning if $A$ starts the game?

Solution. If the first $n-3$ tosses are not heads, neither player is closer to victory, so it is sufficient to join the game when a series of $n-3$ heads has appeared. In this case, the problem is the same as the previous one.

We may encounter a problem that cannot be handled combinatorically at first glance. Here a flowsheet can help us as well, because it can help us 'visualize' the essential features of the problem.

\section{9. 'Passing of the heads' game}

Problem 15. A and $B$ play with three coins. After tossing all three coins simultaneously, the ones with heads goes to the other player. The winner is the 
one who has all three coins. (In the starting case, when $A$ had all three coins, this one is of course ignored.) What is the probability of $A$ winning if $A$ starts the game?

Solution. Look at the flowsheet (Figure 13).

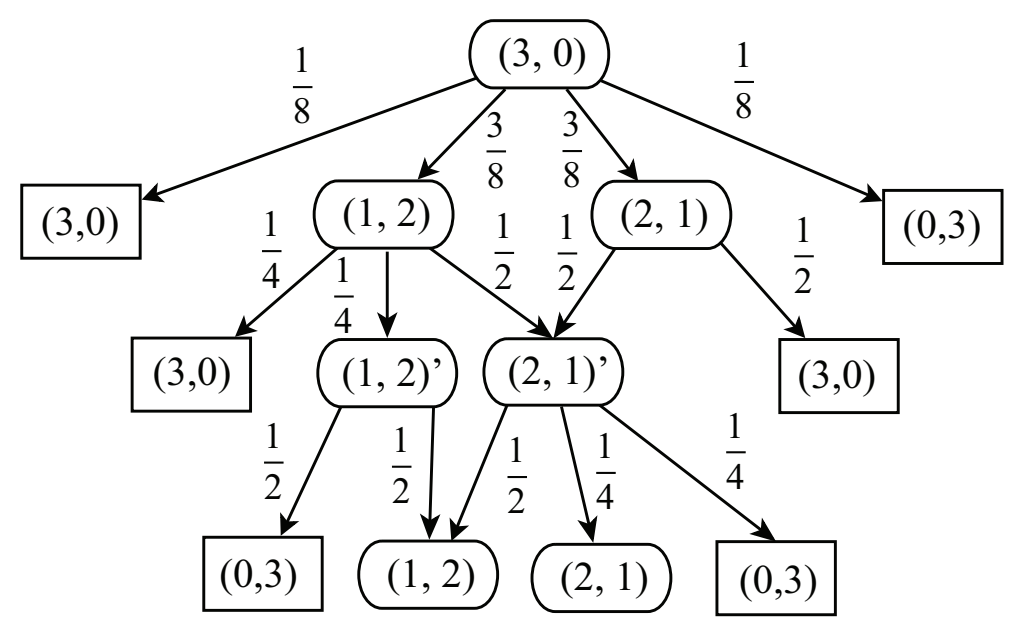

Figure 13. Passing of heads

The $(a, b)$ state means that $A$ has $a$ coins, while $B$ has $b$ coins. Noting the the fact that some states that look the same on the flowsheet are different, it actually does not matter who comes next. In order to simplify matters, let us introduce the notation $(3,0)=x$ (just for the first state), $(1,2)=y,(2,1)=z,(1,2)^{\prime}=u$, $(2,1)^{\prime}=v$ for the "not winning" cases. The $(3,0)$ case represents the starting state, $B$ comes after the states without a prime, and $A$ comes after the states with a prime. (The two conditions are not the same, however. In the first case, the second term tells us the number of coins that are given to the other player. But in the second case, the first term tells us the number of coins that are given to the other player.) The system of equations for this problem is the following:

$$
\begin{aligned}
& P_{x}(A)=\frac{1}{8}+\frac{3}{8} P_{y}(A)+\frac{3}{8} P_{z}(A) \\
& P_{y}(A)=\frac{1}{4}+\frac{1}{4} P_{u}(A)+\frac{1}{2} P_{v}(A) \\
& P_{z}(A)=\frac{1}{2}+\frac{1}{2} P_{v}(A)
\end{aligned}
$$




$$
\text { "mader" — 2008/7/31 — 11:38 — page 38 — \#24 }
$$

$$
\begin{aligned}
& P_{u}(A)=\frac{1}{2} P_{y}(A) \\
& P_{v}(A)=\frac{1}{2} P_{y}(A)+\frac{1}{4} P_{z}(A)
\end{aligned}
$$

The solution in this case is $P_{x}(A)=\frac{53}{88}, P_{y}(A)=\frac{6}{11}, P_{z}(A)=\frac{8}{11}, P_{u}(A)=\frac{3}{11}$, $P_{v}(A)=\frac{5}{11}$.

The game may be interrupted by unforeseen circumstances at any time. One question which arises is the following:

Problem 16 (The problem of fair distribution). How should the winnings be distributed if the game ends at a given point?

Remark. What we think of when we talk about fair distribution is not selfevident. This question should also be discussed with the students in the class before starting.

Solution. By fair distribution at a given point in a game, we mean the distribution of the pot in the ratio of the probability of the players' winnings in a particular state.

Since our flowsheet tells us the probability of a player winning for each given state, the problem of fair distribution can be handled easily as well.

Now let us look at an example connected with the previous exercise.

Suppose the game breaks off at a point and the game ends in state $z$. Then the relative probability of $A$ and $B$ winning is $8: 3$. So they should share the winnings between them in this proportion.

\section{The lazy cashier}

Problem 17. People queue in front of a cashier in order to buy tickets for 50 HUF each. The customers each have a coin of 50 HUF or a coin of 100 HUF with $\frac{1}{2}-\frac{1}{2}$ probability. The lazy cashier did not bring any change with him. What is the probability that at least $n$ people can buy a ticket?

Solution. Let us look at the flowsheet given below (Figure 14). In the state $S$ the cashier's office is empty. The first customer has a coin of 100 HUF with a 


$$
\text { "mader" — 2008/7/31 — 11:38 — page } 39 \text { - \#25 }
$$

probability of $\frac{1}{2}$; in this case the cashier fails to give back change so we arrive at state $F$. The first customer on the other hand may also have a coin of 50 HUF with the probability of $\frac{1}{2}$ as well, which leads us to the state $C_{1}$, and means that the cashier has 50 HUF to go on with. From this point on the flowsheet can be easily continued.

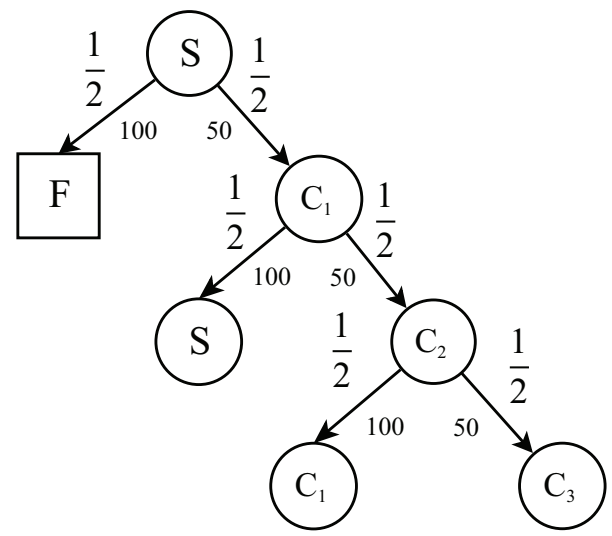

Figure 14. The lazy cashier

With the aid of the flowsheet, the system of equations can also be written down based on the above reasoning.

$$
\begin{aligned}
P_{S} & =\frac{1}{2} P_{C_{1}} \\
P_{C_{1}} & =\frac{1}{2} P_{S}+\frac{1}{2} P_{C_{2}} \\
P_{C_{2}} & =\frac{1}{2} P_{C_{1}}+\frac{1}{2} P_{C_{3}} \\
\vdots & \\
P_{C_{n-2}} & =\frac{1}{2} P_{C_{n-3}}+\frac{1}{2} P_{C_{n-1}} \\
P_{C_{n-1}} & =\frac{1}{2}+\frac{1}{2} P_{C_{n-2}}
\end{aligned}
$$




$$
\text { "mader" — 2008/7/31 — 11:38 — page } 40 \text { — \#26 }
$$

After adding the equations together and rearranging them we get $P_{S}+P_{C_{n-1}}=1$, which means that $P_{C_{n-1}}=1-P_{S}$. Substituting this back into the last but one equation $P_{C_{n-2}}=1-2 P_{S}$, and so on. After the last substitution we get $P_{S}=1-n P_{S}$, thus $P_{S}=\frac{1}{n+1}$.

\section{Summary}

In the above examples we saw how straightforward it was to represent certain discrete probability problems via flowsheets, a method that is currently not in widespread use in secondary education for solving problems involving probability. In experiments some of these problems contain infinitely many recurrences. These type of problems could only be solved with an infinite series, which required a preliminary knowledge of the topic, and this could only be taught at the upper level. Working with flowsheets appears to be useful from several points of view. Some of these are that

- Flowsheets can easily be constructed, and they may also make us rethink the process of the game.

- Probability problems represented as linear systems of equations and linear recursion relations can be described in terms of flowsheets. This approach makes it possible for a wider group of students to tackle such problems and find solutions based on systematic reasoning.

- Flowsheets allow one to visualize the structure of a game task.

- Flowsheets show the content of the problems in question more clearly than most other methods.

- Flowsheets make it possible to easily demonstrate the game.

- It is possible to give the probability of someone winning for each and every state.

- The introduction of conditional probability arises naturally in the flowsheet approach.

- The problem of fair distribution can also be easily solved at any point in time.

- Flowsheets also make it possible to calculate the number of steps that are needed for someone to win and for the game to terminate.

- Probability problems that cannot be handled combinatorically can be readily solved with the aid of flowsheets. 


$$
\text { "mader" — 2008/7/31 — 11:38 — page } 41 \text { — \#27 }
$$

- The playful aspect of flowsheets can motivate students as well, and help them focus on the problem-solving task, working in groups or on their own.

\section{References}

[1] A. Ambrus, Bevezetés a matematika-didaktikába, ELTE Eötvös Kiadó, Budapest, 2004.

[2] W. Feller, An introduction to probability theory and its applications, I, Wiley and Sons-Chapman and H, New York-London, 1950.

[3] J. Kosztolányi, G. Makay, K. Pintér and L. Pintér, Matematikai problémakalauz I., Polygon, Szeged, 1999.

[4] T. Nemetz, Valószinüségszámítás, Tankönyvkiadó, Budapest, 1986.

[5] T. Nemetz and G. Wintsche, Valószínüségszámítás és statisztika mindenkinek, Polygon, Szeged, 1999.

[6] T. M. Mills, Problems in Probability, World Scientific Publishing Co. Pte. Ltd., New Jersey, 2001.

[7] A. Plaza, Proof without words: Partial sum and sum of a geometric series, Teaching Mathematics and Computer Science 2, no. 2 (2004), 423-423.

ATTILA MÁDER

UNIVERSITY OF SZEGED

BOLYAI INSTITUTE

H-6720, SZEGED

ARADI VÉRTANÚK TERE 1.

HUNGARY

SZTE SÁGVÁRI ENDRE HIGHSCHOOL

H-6722, SZEGED

SZENTHÁROMSÁG U. 2.

HUNGARY

E-mail: madera@math.u-szeged.hu

(Received June, 2007) 